Research Article

www.jestr.org

\title{
Multi-Objective Transmission Network Planning with Consideration of Power Grid Vulnerability and Wind Power Accommodation
}

\author{
Chun-guang Tian*, Xiang-yu Lu, Li-sen Chu, Tian Dong and De-xin Li \\ State Grid Jilin Electric Power Company Limited Electric Power Research Institute, \\ Jilin Changchun, 130021, China.
}

Received 20 October 2013; Accepted 12 December 2013

\begin{abstract}
This paper proposes an efficient approach for transmission network expansion planning. Three indicators are proposed to evaluate the planning, which is the power grid vulnerability, wind power accommodation and operation cost. Vulnerability is evaluated based on the complex network theory, and wind power accommodation analysis is performed by the rate of abandoned wind power. The optimization of transmission network expansion planning is translated into constraints multi-objective optimization problem. A novel QS-MOWE algorithm based on the improvement quick sort and NSGA-II algorithm has been proposed. The method can be used effectively to study the effect of increasing wind power integration and vulnerability with high wind generation uncertainties. The model and algorithms are applied to calculate a case of 6 units. The results show that the proposed modeling method can provide a useful guidance for planning problems.
\end{abstract}

Keywords: Transmission network planning, Wind power accommodation, Vulnerability, QS-MOWE, Multi-objective optimization.

\section{Introduction}

Transmission network expansion planning (TNEP) is an important component of power system planning. It determines the characteristics and performance of the future electric power network and influences power system operation directly [1]. Generally, TNEP can be classified as static or dynamic transmission planning. How to determine an optimal network scheme is complex task with minimum operation cost and minimum risk, it is a complex task that there are many uncertainties such as load variations, generator rescheduling, and availability of system facilities.

The large projects of renewable energy sources integration have made the TNEP problem even more challenging. The increased uncertainties sources often require new transmission lines to maintain a level of power system security and adequacy $[2,3]$. Some fault may create cascading failure and then the blackout, even the breakdown of electric power system. So the Vulnerability assessment and prevention method is the key to avoid the blackouts.

The network with large proportion of wind power will have more power flow fluctuations. Wind power causes an extra factor of uncertainties for power system operation and planning. Only one operation scenario is unsuitable for the planning with stochastic power output.

The traditional works of the TNEP problems always used the deterministic approaches, [4] presents power system operating profile. The load demand uncertainties mathematical formulation is shown in [5], Fuzzy models reflecting load or generation variation based on a way of decision maker's experience [6]. System reliability and different investment schemes have been presented in [2].

Optimization problem has been widely used to solve the transmission expansion planning problem, a mixed-integer linear formulation is presented to optimality with considers losses and guarantees convergence [7]. [8] describes a new Benders decomposition approach by using a linear (0-1) model . A decomposition technique is used to simulate plan, three criteria are presented, including investment costs, operation costs [9], Tabu search [10] is proposed to be applied to combinatorial problems. A novel differential evolution algorithm (DEA) [11] is applied to the DC power flow-based model. It solves the problems of static and multistage transmission expansion planning. DC model of TNEP is a mixed integer nonlinear programming problem, it is difficult to solve for large-scale real-world transmission networks. Probabilistic methods are now widely used, it includes probabilistic reliability criteria [12], and chance constrained programming [13].

In this paper, three indicators are proposed to evaluate TNEP, which is the power grid vulnerability, capacity of wind power and cost. Based on the mentioned definition, the improvement quick sort model and algorithms of flexible transmission system planning are proposed. The model and algorithms are applied to calculate a case of 6 units. The results show that the proposed modeling method can provide a useful guidance for planning problems.

\footnotetext{
* E-mail address: win_shb@163.com

ISSN: 1791-2377 @ 2011 Kavala Institute of Technology. All rights reserved.
} 


\section{Mathematical Model of Multi-Objective Transmission Network Planning}

Three indicators are proposed to evaluate TNEP, which is the vulnerability, capacity of wind power and operation cost. The first important aspect is power grid vulnerability. Based on complex network theory, the power grid is simplified as a topological model. Generator, load and substation is simplified as a node in the network, the transmission line is the edges in the network. The electric power network is sparse graphs with n-nodes and the k-lines by the connection weight matrix.

The topological model mainly presents two topological parameters, the node betweenness and line betweenness, two efficiency index, which is the most effective path efficiency and global system effectiveness. The most effective path effectiveness $\varepsilon_{i j}$ is the line betweenness sum along the generator nodes I and load nodes $\mathrm{j}$

$\varepsilon_{i j}=\left(\sum \frac{1}{E_{m l}}\right)^{-1}$

Where line $m-l \in S, S$ is line aggregate along path, $\left(E_{m l}\right)_{n \times n}$ is the connection weight matrix, $E_{m l}$ is the circuit $m-l$ effectiveness, The node betweenness $B_{N}$ is node times by the shortest path in the network between all generator nodes and load nodes, line betweenness $B_{L}$ the line betweenness $B_{N}$ is node times by the shortest path in the network between all generator nodes and load nodes.

Global system efficiency $E_{g}$ is efficiency average value of the most effective path between all generator nodes and load nodes in network. It reflects the whole network power transmission capability.

$$
E_{g}=\frac{1}{N_{L} N_{G}} \sum_{i \in N_{l}} \sum_{j \in N_{G}} \varepsilon_{i j}
$$

Where $N_{L}$ is number of system load nodes. $N_{G}$ is number of generator nodes. Line effectiveness is showed as formula (3).

$$
E_{m l}^{\prime}=\left\{\begin{array}{cc}
\frac{e_{m l} C_{m l}}{P_{m l}^{\prime}} & P_{m l}{ }^{\prime}>C_{m l} \\
e_{m l} & P_{m l}^{\prime}<C_{m l}
\end{array}\right.
$$

Where

$$
e_{m l}= \begin{cases}\frac{e_{m l 0} C_{m}}{B_{N m}{ }^{\prime}} & B_{N m}{ }^{\prime}>C_{m} \\ e_{m l 0} & B_{N m}{ }^{\prime}<C_{m}\end{cases}
$$

$P_{m l}^{\prime}$ is active power flow of line $m-l, C_{m l}$ is lines transmission limit, $C_{m l}=\beta \cdot P_{m l 0}, \beta$ is tolerance coefficient, $\beta \mathrm{f} 1 \cdot e_{m l 0}$ is initial line effectiveness of line $m-l$, $B_{N m}{ }^{\prime}$ is the fault node betweenness, $C_{m}$ is the units upper limit, $C_{m}=\alpha \cdot B_{n m 0}, \alpha$ is tolerance coefficient, In this paper, Decline of global effectiveness is used to measure vulnerability of transmission network planning after the fault system.

$$
D_{\mathrm{g}}=\frac{E_{\mathrm{g} 0}-E_{\mathrm{g}}}{E_{\mathrm{g} 0}}
$$

where $D_{\mathrm{g}}$ is decline of global effectiveness. $E_{\mathrm{g} 0}$ is initial global system effectiveness

The second important aspect of power system planning is wind power capability, The expression of wind power capability is shown in $\alpha_{W}$. In this function, $Q_{d}^{w, c}$ is abandoned day wind power, $\alpha_{W}$ is the rate of abandoned wind power:

$$
\begin{aligned}
& \alpha_{W}=\sum Q_{d}^{w, c} / \sum Q_{d}^{w} \\
& Q_{d}^{w, c}=\sum_{t=1}^{T} P_{d, t, m}^{W, c} \\
& P_{d, t, m}^{w, c}=P_{r}\left(E_{0}\right) *\left(P_{d, t, m}^{w}-P_{t, m}^{w, s}\right) \\
& P_{t, d, m}^{w, s}=P_{t, d, m}^{L}-P_{\min , d, m}^{G}
\end{aligned}
$$

where $t$ is various time intervals at typical day, $T$ is the number of period of days, $\mathrm{m}$ is month, $L$ is load, $W$ is wind power, $S$ is receiving space, $P_{t, d, m}^{w, s}$ is each period space to accommodate wind, $P_{\min , d, m}^{G}$ is the minimum value of system power output. $P_{t, d, m}^{L}$ is sequence in time curve, $Q_{d}^{w}$ is day generated electrical energy without abandoned wind power, $P_{r}\left(E_{0}\right)$ is the probability of occurrence of an event.

The constraints of this model mainly include: Active power balance of power system as formula (9), Upper and lower limits of unit output as formula (10), Spinning reserve limit of the system as formula (11), Minimal on/off time duration as formula (12), Ramp rate limit as formula (12).

$$
\sum_{k=1}^{N G} P_{g k}(t)-P_{a b d}(t)=P_{L}(t)
$$

$u_{g t} \cdot P_{k, \min } \leq P_{g k}(t) \leq u_{g k} P_{k, \max }$

$P_{L}(t)\left(1+S_{R}\right) \leq \sum_{k=1}^{N_{G}} u_{g k}(t) P_{k, \max }$

$T_{g k}^{o n}(t) \geq T_{k}^{u p} ; T_{k}^{\text {down }} \leq T_{g k}^{o f f}(t)$

$\left|P_{g k}(t)-P_{g k}(t-1)\right| \leq u_{g k}(t) P_{k}^{r a m p}$ 
where $S_{R}$ is the spinning reserve. $T_{k}^{u p}$ and $T_{k}^{\text {down }}$ is the minimal on/ off time duration; $T_{g k}^{o n}(t)$ and $T_{g k}^{o f f}(t)$ is the accumulated on/off time duration.

The third important aspect of power system planning is the economic evaluation, the annual operation cost is optimization object based on network loss and investment cost. It is shown in formula

$$
\begin{aligned}
C_{p l}= & \sum_{(i, j) \in \Omega} \frac{c_{i j} x_{i j} \cdot r \cdot(1+r)^{Y e a r}}{(1+r)^{Y e a r}-1} \\
& +\gamma \cdot \tau_{i j \max } \cdot \sum_{(i, j) \in \Omega} x_{i j} \cdot R_{i j} \cdot E\left(\left(P_{i j}\right)^{2}\right)
\end{aligned}
$$

Where $c_{i j}$ is investment cost of candidate central line $(i, j), \gamma$ is unit price, $\boldsymbol{\tau}_{i j \max }$ is annual peak load hours, $x_{i j}$ is line Integer decision variables. $r$ is discount rate, year is line economic life, $R_{i j}$ is branch single line Electrical, $E\left(\left(P_{i j}\right)^{2}\right)$ is branch flow square expected value.

The constraints of this model mainly include: System power Equilibrium equation, Flow equation, and the generator output upper and lower limit, new add line channel constraints, the chance constraint of line flow.

$$
\begin{aligned}
& \sum_{j=1, j \neq i}^{N} P_{i j, n}+\sum_{k=1}^{N G_{i}} P_{g k, n}+P_{L i_{-} i, n}=P_{L i, n}+\sum_{j=1}^{N_{i}} P_{a b c, j} \\
& P_{i j}=\left(b_{i j}^{(0)}+x_{i j} b_{i j}\right)\left(\delta_{i}-\delta_{j}\right), \forall(i, j) \in \Omega \\
& P_{g k, n, \min } \leq P_{g k, n} \leq P_{g k, n, \max }, k \in \Psi_{h t} \\
& 0 \leq x_{i j} \leq x_{i j \max }, \forall(i, j) \in \Omega \\
& \operatorname{Pr}\left(P_{i j} \leq x_{i j} \cdot P_{i j \max }\right) \leq 1-\eta_{1} \\
& \operatorname{Pr}\left(P_{i j}^{(N-1)} \leq x_{i j} \cdot P_{i j \max }\right) \leq 1-\eta_{2}
\end{aligned}
$$

\section{Transmission Network Planning Multi-Objective Optimized Model Based on Improvement Quick Sort Algorithms}

Based on the evaluation functions, the multi-objective optimization model is constructed to maximize the satisfactions of different objectives by adjusting TNEP. The multi-objective optimization model is represented as:

$$
\operatorname{Min}\left(\operatorname{Mep}\left(D_{\mathrm{g}}\right), \operatorname{Mep}\left(\alpha_{W}\right), \operatorname{Min}\left(\operatorname{Mep}\left(C_{p l}\right)\right)\right.
$$

$$
\begin{aligned}
& \text { Subject to } \\
& \sum_{j=1, j \neq i}^{N} P_{i j, n}+\sum_{k=1}^{N G_{i}} P_{g k, n}+P_{L i_{-} i, n}=P_{L i, n}+\sum_{j=1}^{N_{i}} P_{a b c, j} \\
& P_{g k, n, \min } \leq P_{g k, n} \leq P_{g k, n, \max }, k \in \Psi_{h t}
\end{aligned}
$$

$$
\begin{gathered}
P_{L}(t)\left(1+S_{R}\right) \leq \sum_{k=1}^{N_{G}} u_{g k}(t) P_{k, \text { max }} \\
P_{i j}=\left(b_{i j}^{(0)}+x_{i j} b_{i j}\right)\left(\delta_{i}-\delta_{j}\right), \forall(i, j) \in \Omega \\
0 \leq x_{i j} \leq x_{i j \max }, \forall(i, j) \in \Omega \\
\operatorname{Pr}\left(P_{i j} \leq x_{i j} \cdot P_{i j \max }\right) \leq 1-\eta_{1} \\
\operatorname{Pr}\left(P_{i j}^{(N-1)} \leq x_{i j} \cdot P_{i j \max }\right) \leq 1-\eta_{2}
\end{gathered}
$$

The aim of TNEP multi-objective optimization is find a solution. In this formula, $\operatorname{Mep}\left(\alpha_{W}\right), \operatorname{Mep}\left(D_{\mathrm{g}}\right), \operatorname{Mep}\left(C_{p l}\right)$ is objective function with minimum operation cost, risk and abandoned wind power rate. The constraint function is very complex for the big search space, There are many algorithms, like SPEA2 [14], PESA - II [15], NGSA - II [16] and Micro-GA [17], but the NGSA-II algorithm divides the population in fronts of non-dominated solutions, the interesting search space can be addressed, where the global pareto-optimal region is located. We presented several concepts as follows:

Definition 1: (Pareto Dominance): A vector $u=\left(u_{1}, u_{2}, \ldots u_{n}\right)$ is said to dominate $v=\left(v_{1}, v_{2}, \ldots v_{n}\right)$, iff $\mu$ is partially less than $v, \forall i \in\{1, . ., n\}, u i \leq$. $v i \wedge \exists i \in\{1, \ldots, n\}: u i<v i$

Definition 2: (Pareto Optimality): A solution $x \in X$ is pareto optimal with respect to $\mathrm{X}$ iff not $x^{\prime} \in X$ for which $v=f\left(x^{\prime}\right)=\left(f_{1}\left(x^{\prime}\right), \ldots ., f_{n}\left(x^{\prime}\right)\right)$ dominates $u=f(x)=\left(f_{1}(x), \ldots, f_{n}(x)\right)$.

Definition 3: $\mathrm{X}$ is a non-dominated individual, and $\mathrm{Y}$ is a dominated individual, $X \succ Y$, “ $\succ$ " denotes the dominatedrelation. $\forall X, Y \in P o p, \mathrm{X}$ relates with $\mathrm{Y}$, iff $X \succ Y$ or $\mathrm{X}=$ $\mathrm{Y}$; otherwise there is no relation between $\mathrm{X}$ and $\mathrm{Y}$

Definition 4: $\forall X, Y \in P o p \quad X>_{d} Y$ iff $X \succ Y$ or $\mathrm{X}$ does not relate with $Y$. A partial order $\geq_{n}$ is defined as, $i_{\text {rank }}$ represents Non-domination rank, and $i_{\text {distance }}$ represents local crowding distance. $\quad \mathrm{I} \geq_{\mathrm{n}} \mathrm{j}$ if $\left(\mathrm{i}_{\text {rank }}<\mathrm{j}_{\text {rank }}\right)$ or $\left(\left(\mathrm{i}_{\text {rank }}=\mathrm{j}_{\text {rank }}\right)\right.$ and $\left.\left(\mathrm{i}_{\text {distance }}>\mathrm{j}_{\text {distance }}\right)\right)$.

We proposed the novel algorithm (QS-MOWE) based on the improvement quick sort [18] and NSGA-II algorithm. Suppose that the size of population $P$ is $n$, and $P_{t}$ is the $\mathrm{t}$ generation population. $Q_{t}$ is a new evolutionary population from $P_{t}$ by the updated selection, crossover and mutation operators. Let $R_{t}=P_{t} \mathrm{U} Q_{t}$, and the size of $R_{t}$ is $2 \mathrm{n}$. The non-dominated set $P_{1}$ is generated from $R_{t}$, with the quick sort procedure. If $|P 1| \mathrm{f} n$, the clustering procedure reduces the size $P_{1}$, and to keep the diversity of $P_{1}$, the size of $P_{1}$ will be $n$ after the clustering process.

\section{Algorithm 1. QS-MOWE Algorithm}

$P t, t=0 ; / /$ set $\quad t=0$.

Generate an initial population $\mathrm{P}[\mathrm{t}]$ 
While $(\mathrm{t} \leq \mathrm{T})$

do

$/ \mathrm{T}$ is max number of generations

$\left\{\mathrm{Q}_{\mathrm{t}}=\right.$ make - new $-\operatorname{pop}\left(\mathrm{P}_{\mathrm{t}}\right)$

// Use selection, crossover and mutation to create a new

populationQ

$\mathrm{R}_{\mathrm{t}}=\mathrm{P}_{\mathrm{t}} \cup \mathrm{Q}_{\mathrm{t}}$

// Combine parent and

children population

$\mathrm{P}_{\mathrm{t}+1}=\operatorname{NDSet}\left(\mathrm{R}_{\mathrm{t}}\right)$

/ / construct non - dominated

set with quick sort

If $\left(\left|\mathrm{P}_{\mathrm{t}+1}\right|<=\mathrm{N}\right)$

Then $\left\{\mathrm{P}_{\mathrm{t}+1}=\mathrm{P}_{\mathrm{t}+1} \cup\right.$ select -

by $-\operatorname{random}\left(\mathrm{R}_{\mathrm{t}}-\mathrm{P}_{\mathrm{t}+1}, \mathrm{~N}-\left|\mathrm{P}_{\mathrm{t}+1}\right|\right)$

/ / randomly selectedN -

$\left|P_{t+1}\right|$ elements and joined into $P_{t+1}$

Else if $\left(\left|\mathrm{P}_{\mathrm{t}+1}\right|>\mathrm{N}\right)$

Then \{crowding - distance -

assignment $\left(\mathrm{P}_{\mathrm{t}+1}\right)$

//Calculate crowding distance.

Sort $\left(\mathrm{P}_{\mathrm{t}+1}, \mathrm{z}_{\mathrm{n}}\right)$

// Sort in descending order using $\geq_{n}$

$\left.\mathrm{P}_{\mathrm{t}+1}=\mathrm{P}_{\mathrm{t}+1}[1: \mathrm{N}]\right\}$

/ / Choose the first $\mathrm{N}$ elements

\section{Algorithm NDSet}

\section{Function NDSet}

(Var Pop : population; $s, t$ : int eger)

$\{$ NDSet $=$;

$\mathrm{i}=\mathrm{s} ; \mathrm{j}=\mathrm{t} ; \mathrm{X}=\operatorname{pop}[\mathrm{s}]$;

While $>2$ do

$($ non - dominated - sign $=. T$;

while $(\mathrm{i}<\mathrm{j})$ do

( while $(\mathrm{i}<\mathrm{j})$ and (Xpop $[\mathrm{i}]$ ) do $\mathrm{j}=\mathrm{j}-1$;

$\mathrm{p}=\operatorname{Pop}[\mathrm{i}] ; \operatorname{Pop}[\mathrm{i}]=\operatorname{pop}[\mathrm{j}] ; \operatorname{pop}[\mathrm{j}]=\mathrm{p}$;

while $(\mathrm{i}<\mathrm{j})$ and $\left(\operatorname{Pop}[\mathrm{j}]>_{\mathrm{d}} \mathrm{X}\right)$ do

( $\mathrm{i}=\mathrm{i}+\mathrm{l}$;

if $(\operatorname{Pop}[\mathrm{i}] \mathrm{X})$ then non - dominated - sign $=. \mathrm{F}$. $)$

$\mathrm{p}=\operatorname{Pop}[\mathrm{i}] ; \operatorname{Pop}[\mathrm{i}]=\operatorname{Pop}[\mathrm{i}] ; \operatorname{Pop}[\mathrm{j}]=\mathrm{p}$;

\}

If (non - dominated - sign) then

NDSet $=$ NDSet

$\mathrm{j}=\mathrm{i}-1 ; \mathrm{i}=\mathrm{s} ; \mathrm{X}=\operatorname{Pop}[\mathrm{s}]$;)

\} end for function
The function NDSet () returns a non-dominated set. The time complexity can be proved that Algorithm NDSet () is less than $O(n \log n)$. It is better than $O\left(n^{2}\right)$ in the NSGAII.

\section{Case Study}

The Garver [19] test system has six buses, 15 candidate branches, and a total demand equal to $760 \mathrm{MW}$. Reactive power demand in each bus is assumed to be $10 \%$ of the active power demand in that bus. We assume every generator submits its supply offer in the form of a linear function. Table1 shows the existing and candidate lines data.

The problem has 1762 single equations, 1189 single variables and 15 discrete variables. Among all 49 subproblems, 12 sub-problems are infeasible. Therefore the remaining 37 sub-problems have integer solution or are locally optimal. A power benchmark value is 100MVA, voltage benchmark value is $220 \mathrm{kV}, \mathrm{R}$ is $10 \%, \mathrm{n}$ is $15 \mathrm{a}, \tau$ is $3000 \mathrm{~h}, \rho$ is 0.3 yuan/(kWh), $C_{j}$ is 1000 thousands yuan $/ \mathrm{km}$. The tolerance coefficient $\alpha, \beta$ is 1.05 , Annual loss hours is $2000 \mathrm{~h}$.

Table1. Lines Data

\begin{tabular}{c|c|c|c|c|c|c}
\hline From & To & Capacity & Length & Resistance & Reactance & Operation \\
\hline 1 & 4 & 80 & 60 & 0.15 & 0.6 & --- \\
1 & 5 & 100 & 20 & 0.05 & 0.20 & --- \\
2 & 3 & 100 & 20 & 0.05 & 0.20 & --- \\
2 & 4 & 100 & 40 & 0.10 & 0.40 & --- \\
3 & 5 & 100 & 20 & 0.05 & 0.2 & -- \\
1 & 2 & 100 & 40 & 0.10 & 0.40 & 40 \\
1 & 3 & 100 & 38 & 0.09 & 0.38 & 38 \\
1 & 4 & 80 & 60 & 0.15 & 0.60 & 60 \\
1 & 5 & 100 & 20 & 0.05 & 0.20 & 20 \\
1 & 6 & 70 & 68 & 0.17 & 0.68 & 68 \\
2 & 3 & 100 & 20 & 0.05 & 0.20 & 20 \\
2 & 4 & 100 & 40 & 0.10 & 0.40 & 40 \\
2 & 5 & 100 & 31 & 0.08 & 0.31 & 31 \\
2 & 6 & 100 & 30 & 0.08 & 0.30 & 30 \\
3 & 4 & 82 & 59 & 0.15 & 0.59 & 59 \\
3 & 5 & 100 & 20 & 0.05 & 0.20 & 20 \\
3 & 6 & 100 & 48 & 0.12 & 0.48 & 48 \\
4 & 5 & 75 & 63 & 0.16 & 0.63 & 63 \\
4 & 6 & 100 & 30 & 0.08 & 0.30 & 30 \\
5 & 6 & 78 & 61 & 0.15 & 0.61 & 61 \\
\hline
\end{tabular}

Table2. Result Comparison of 6 Node Case

\begin{tabular}{|c|c|c|c|c|}
\hline $\begin{array}{l}\text { Objective and } \\
\text { Methods }\end{array}$ & $\alpha_{W}$ & $D_{\mathrm{g}}$ & $C_{p l}$ & New lines Circuit \\
\hline $\begin{array}{c}\operatorname{Min}\left(\alpha_{W}, D_{\mathrm{g}}, C_{p l}\right) \\
(\mathrm{Q}-\mathrm{MOWE})\end{array}$ & 3.4 & 72.3 & 16070 & $\begin{array}{l}3-5(2), 2-6(4), \\
4-6(3)\end{array}$ \\
\hline $\operatorname{Min}\left(\alpha_{W}\right)$ & 0 & 79.3 & 19276 & --- \\
\hline $\operatorname{Min}\left(D_{\mathrm{g}}\right)$ & 7.2 & 70.4 & 81600 & --- \\
\hline $\operatorname{Min}\left(C_{p l}\right)$ & 7.6 & 73.2 & 14040 & ---- \\
\hline $\operatorname{Min}\left(D_{\mathrm{g}}, \alpha_{w}\right)$ & 3.3 & 72.5 & 95400 & $\begin{array}{c}3-5(2), 2-6(4), 4- \\
6(3), \\
3-6(1)\end{array}$ \\
\hline
\end{tabular}

Tables 2 presents the results obtained using the algorithm proposed in this paper for different Objective. It shows the relationship of vulnerability, abandon wind rate and risk rate by multi-objective optimization. With 
considering $\alpha_{W}, D_{g}, C_{p l}$ separately and two goals $\left(D_{\mathrm{g}}, C_{p l}\right)$, The results of data is different. The $\alpha_{W}$ is $0, D_{\mathrm{g}}$ is 79.3 and $C_{p l}$ is 12276 in the Min $\left(\alpha_{W}\right)$ model, the $\alpha_{W}$ is 7.2, $D_{\mathrm{g}}$ is 70.4 and $C_{p l}$ is 81600 in the $\operatorname{Min}\left(D_{\mathrm{g}}\right)$ model, the $\alpha_{W}$ is 7.6, and $D_{\mathrm{g}}$ is $73.2 C_{p l}$ is 10404 in the Min $\left(C_{p l}\right)$ model. Compare with various indicators of Multi objective model, It show that the single objective model is not economical and not balance multiple target value, It can be seen that the optimal value of two goals $\left(D_{\mathrm{g}}, C_{p l}\right)$ is the $\alpha_{W}$ is 3.3, and $D_{\mathrm{g}}$ is $72.5 C_{p l}$ is 95400 , and the QS-MOWE model is $3.4\left(\alpha_{W}\right), 72.3\left(D_{\mathrm{g}}\right) \cdot 16070\left(C_{p l}\right)$.This method has obvious advantages in the economy, and it balanced the multiobjective optimization problem in transmission network planning.

With the increase of abandoned wind power, the influence of wind speed random is being reduced to operation cost and system risk gradually. To reduce the Wind Power Accommodation can effectively decrease the risk and operation cost.

\section{Conclusion}

Transmission network expansion planning is an important component of power system planning. The large integration of renewable energy sources has made the TNEP problem even more challenging. Some fault in local grid could be extended and transmitted quickly, so the risk assessment for cascading failures is the key to avoid the blackouts. An efficient approach for transmission network expansion planning is proposed. Three indicators are proposed to evaluate TNEP, which is the power grid vulnerability, capacity of wind power and operation cost. To evaluate the electricity network transmission ability and withstanding capacity of failures, the transmission network vulnerability index takes into account the structural characteristics and operation states. The optimization of TENP is translated into constraints multi-objective optimization problem. A novel QS-MOWE algorithm based on the improvement quick sort and NSGA-II algorithm has been proposed. The method can be used effectively to study the effect of increasing wind power integration and vulnerability with high wind generation uncertainties. The model and algorithms are applied to calculate a case of 6 units. The results show that the proposed modeling method can provide a useful guidance for planning problems.

\section{Acknowledgements}

This work was supported in part by:

(1) Project Supported by Scientific Research Fund of Jilin Provincial Education (20120268).

(2) A Project Supported by Scientific and Technological Planning Project of Jilin Province (20120332).

(3)A Project Supported by Scientific and Technological Planning Project of Jilin Province (20100565).

\section{References}

1. George A, Pavlos S, Transmission expansion planning of systems with increasing wind power integration, IEEE Transactions on Power Systems, 2013, 28(2):1355-1362.

2. Bouffard F, Galiana F.D, Stochastic security for operations planning with significant wind power generation. IEEE Trans. Power Syst, 2008, 22(1):306-316.

3. Billinton R, Wangdee W, Reliability-based transmission reinforcement planning associated with large-scale wind farms," IEEE Trans. Power Syst,2007, 22(1):34-41.

4. Rahmani M, Rashidinejad M, Carreno E.M, Efficient method for AC transmission network expansion planning, Electric Power Systems Research, 2010,80(9): 1056-1064.

5. De I, Silva J, Rider M. J, Romero R, Transmission network expansion planning considering uncertainty in demand, IEEE Trans. Power Syst, 2006, 21(4):1565-1573.

6. Jaeseok C, El-Keib A. A, Tran T, A fuzzy branch and bound based transmission system expansion planning for the highest satisfaction level of the decision maker, IEEE Trans. Power Syst, 2005, 20(1): 476-484.

7. Alguacil N, Motto A. L, Conejo A. J, Transmission expansion planning: A mixed-integer LP approach, IEEE Trans. Power Syst,2003, 18(3):1070-1077.

8. Binato S, Pereira M. V. F, Granville S, A new Benders decomposition approach to solve power transmission network design problems, IEEE Trans. Power Syst, 2001, 16(2): 235-240.

9. Braga A. S. D, Saraiva J. T, A multiyear dynamic approach for transmission expansion planning and long-term marginal costs computation, IEEE Trans. Power Syst, 2005, 20(3):1631-1639.

10. E. L, Silva Da, Ortiz J. M. A, Oliveira G. C, Binato S, Transmission network expansion planning under a tabu search approach, IEEE Trans. Power Syst, 2001,16(1):62-68.

11. Sum-Im T, Taylor G. A, Irving M. R, Song Y. H, Differential evolution algorithm for static and multistage transmission expansion planning, IET Gen, Transm, Distrib, 2009, 3(4): 365384.
12. Choi J, Tran T, El-Keib A. A, Thomas R, A method for transmission system expansion planning considering probabilistic reliability criteria, IEEE Trans. Power Syst, 2005, 29(3):1606-1615.

13. $\mathrm{Yu} \mathrm{H}$, Chung $\mathrm{C}$. $\mathrm{Y}$, Wong $\mathrm{K}$. P. A chance constrained transmission network expansion planning method with consideration of load and wind farm uncertainties, IEEE Transactions On Power Systems, 2009, 24(3): 1568-1576.

14. Hui L, Zhang Q.F,Multi-objective optimi-zation problems with complicated pareto sets, MOEA/D and NSGA-II, IEEE Transactions on Evolutionary Computation, 2009,13(2): 284302.

15. Corne D.W, Jerram N.R, Knowles J.D, PESA-II: regionbased selection in evolutionary multiobjective optimization. In: Proceedings of the Genetic and Evolutionary Computation Conference, 2001: 283-290.

16. Deb K, Pratap A , Agrawal S. A Fast and Elitist Multi objective Genetic Algorithm: NSGA-II. IEEE Transactions on Evolutionary Computation, 2002, 6(2):182 - 197.

17. Carlos A, Coello C, Pulido G.T. Multi-objective Structural Optimization using a Micro-Genetic Algorithm, Structural and Multidisciplinary Optimization, 2005, 30(5): 388-403.

18. Zheng J.H Ling C.X, Shi Z.Z, Xie Y .Some discussions about MOGAs: individual relations, non-dominated set, and application on automatic negotiation. Evolutionary Computation, 2004, 1: 706- 712

19. Romero R, Monticelli A, Garcia A, Haffner S, Test systems and mathematical models for transmission network expansion planning, IEEE Proceedings Generation, Transmission and Distribution, 2002, 149(1): 27-36. 